\title{
Magnetic Resonance Imaging of Non-Puerperal Complete Uterine Inversion
}

\author{
Veli Mihmanli ${ }^{1}$; Fahrettin Kilic ${ }^{2, *}$; Soner Pul ${ }^{1}$; Aydin Kilinc ${ }^{1}$; Ahmet Kilickaya ${ }^{1}$ \\ ${ }^{1}$ Department of Obstetrics and Gynecology, Okmeydani Training and Research Hospital, Istanbul, Turkey \\ ${ }^{2}$ Department of Radiology, Medical Faculty, Istanbul University Cerrahpasa, Istanbul, Turkey \\ ${ }^{*}$ Corresponding author: Fahrettin Kilic, Department of Radiology, Medical Faculty, Istanbul University Cerrahpasa, Istanbul, Turkey. Tel: +90-2124143000; Fax: +90-5325004879, \\ E-mail: fahrettinkilic@hotmail.com
}

Received: December 20, 2012; Revised: June 7, 2013; Accepted: October 27, 2013

\begin{abstract}
Uterine inversion is shortly described as the indentation and depression of the fundic area extending downwards up to the different levels of the birth canal till vaginal opening. Clinical diagnosis of uterine inversion is difficult due to its non-specific symptoms and physical examination. Ultrasonography is the most practical modality for radiological evaluation, but it is inadequate to determine the exact nature of this condition and making the differential diagnosis. In this case, we present the main MRI findings of non-puerperal complete uterine inversion caused by a giant leiomyoma.
\end{abstract}

Keywords: Uterine Inversion; Uterine Prolapse; Magnetic Resonance; Ultrasonography; Uterine Diseases

\section{Introduction}

Uterine inversion is shortly described as the indentation and depression of the fundic area extending downwards up to the different levels of the birth canal till the vaginal opening. It is a rare entity experienced in two different conditions; namely, puerperal (obstetric) or non-puerperal (gynecologic). Non-puerperal inversion occurs less frequently limited to case reports and it is associated with the traction effect of mass lesions, mostly benign submucous myomas, attached to the fundus (1, 2). Clinical diagnosis of uterine inversion is difficult. Imaging modalities are essential for definite diagnosis and afterwards management. This report mainly presents the magnetic resonance imaging (MRI) findings of a complete uterine inversion caused by a giant leiomyoma.

\section{Case Presentation}

A 47-year-old peri-menopausal woman presented with bilateral groin pain, inability to urinate, and bloody vaginal discharge. Clinical vaginal examination revealed the presence of a large necrotic mass filling the whole vaginal tract. Fundus of the uterus was not palpable. Bleeding from the uterus continued excessively. The transabdominal ultrasound examination showed a cervico-vaginal mass measuring $112 \times 114 \mathrm{~mm}$ (Figure 1) that protruded from the uterine cavity with the distorted view of the uterus. After consultation, we considered the possibility of uterine inversion with an unknown nature of the accompanying mass and MRI examination was ordered. MRI confirmed a large heterogeneous signal intensity of well-defined submucosal fundal fibroid mass extending till vaginal opening and sinking of the uterine fundus getting close to the upper vagina. Uterine anatomy was distorted as seen as "V shape" fundus in sagittal plane due to traction of the mass (Figure 2). There was no evidence of adjacent organ involvement within the disease, nor lymph node enlargement. Laboratory tests revealed serum Hb:10.1 g/dL, hematocrit: $30.9 \%$, CRP: $37.5 \mathrm{mg} / \mathrm{L}$, fasting glucose: $86 \mathrm{mg} / \mathrm{dL}, \mathrm{AST}$ : $29 \mathrm{U} / \mathrm{L}, \mathrm{ALT}: 26 \mathrm{U} / \mathrm{L}$, and CA125:152 U/mL.

Laparotomy was performed through midline incision. At laparotomy, a large uterine mass with complete uterine inversion was confirmed (Figure 3). Bladder was elevated due to mass compression and fundus was inverted totally. Primarily, we tried to take out the mass through the vagina, but it was not possible because of the size of the mass. Both fallopian tubes and ovaries were in the pelvis and located outside the concavity of the inverted uterus. The mass was taken out via transverse uterine incision after depicting the myoma's pedicle. Total abdominal hysterectomy and bilateral salpingo-oophorectomy was performed due to the patient's request before surgery. The excised specimen revealed a giant mass with extensive necrosis and multiple hemorrhagic foci. Pathology report confirmed $12 \times 12 \times 10 \mathrm{~cm}$ sized leiomyoma including large ischemic necrotic areas (Figure 4). There was no evidence of malignancy. The patient was discharged without any complications five days after the surgery.

Copyright ( ) 2015, Tehran University of Medical Sciences and Iranian Society of Radiology. This is an open-access article distributed under the terms of the Creative Commons Attribution-NonCommercial 4.0 International License (http://creativecommons.org/licenses/by-nc/4.0/) which permits copy and redistribute the material just in noncommercial usages, provided the original work is properly cited. 


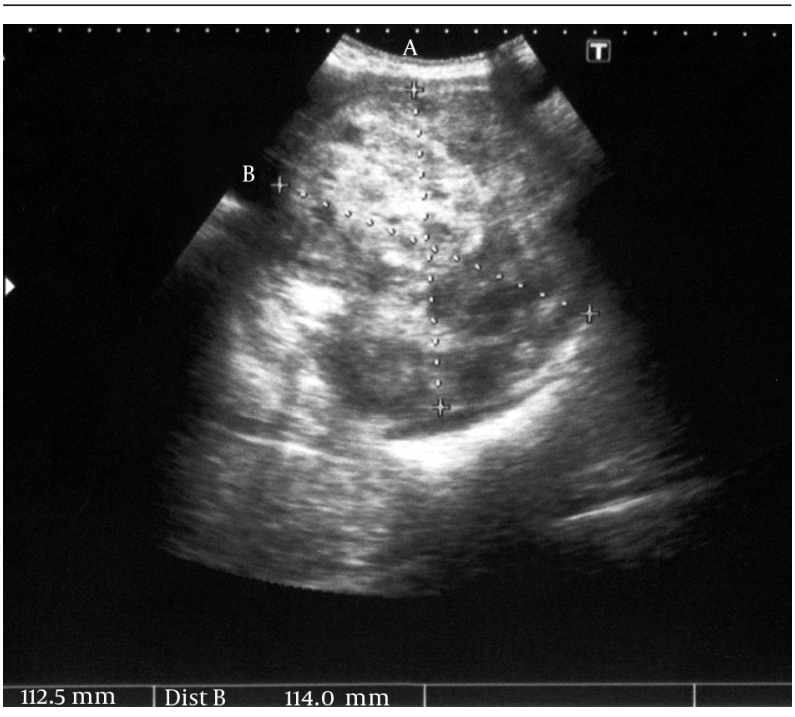

Figure 1. A 47-year-old perimenopausal woman with uterine inversion. Longitudinal transabdominal gray scale ultrasound image shows heterogeneous echo textured well-defined mass lesion measured $112 \times 114 \mathrm{~mm}$ at the cervico-vaginal level. Uterine morphology is not obvious because of the giant mass.

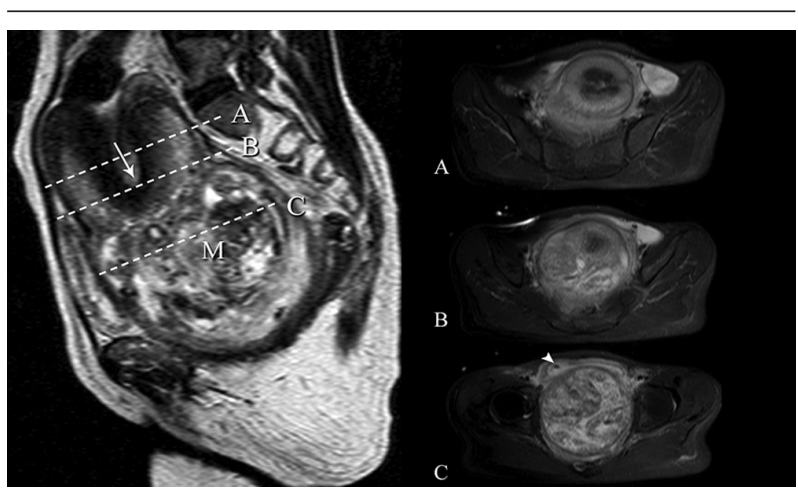

Figure 2. Sagittal T2-weighted MRI shows a heterogeneous leiomyoma mass (M) obliterating the lower pelvis. There is no anatomic correlation of cervix and vagina. The normal anatomy of the uterus is not recognizable. Uterine anatomy was distorted as seen as "V shape" fundus (arrow) due to traction of the mass. In axial slices with fat saturated T2-weighted images (A), section through the upper (B), and more caudal level shows layers of the inverted uterus with "bull's-eye" configuration (C), More caudal axial image shows the heterogeneous leiomyoma mass. Mass compresses the bladder with urinary catheter (arrowhead). Minor pelvic effusion is present.

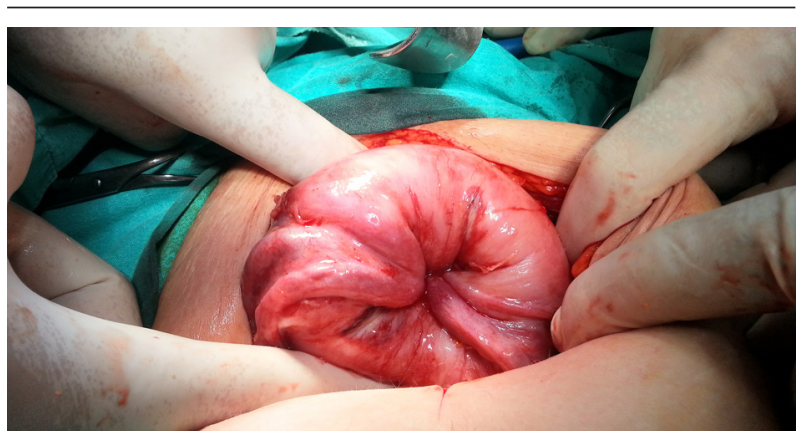

Figure 3. Uterine inversion found at laparotomy. Note the minor ischemic color changes in the inverted portion.

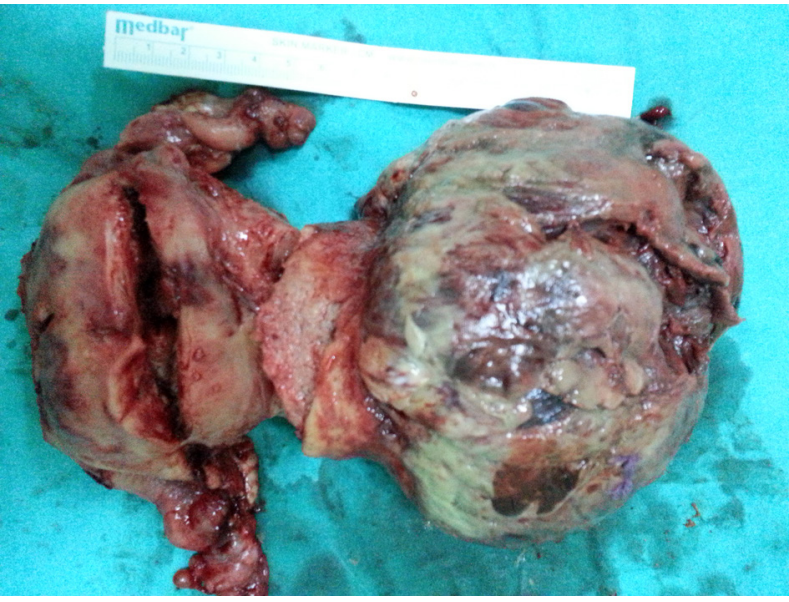

Figure 4. The incised uterus and prolapsed giant leiomyoma

\section{Discussion}

Non-puerperal uterine inversion is a rare condition that can be presented due to gravitational mass effect of tumors. Rare causes include uterine tumors such as sarcomas, carcinomas and mostly benign conditions such as leiomyomas as in our case $(1,2)$. Diagnosis of non-puerperal uterine inversion can be difficult as they may not present with common typical symptoms (3). Clinical differential diagnosis of uterine inversion is troublesome even if the fundal depression can be palpated on rectal examination because of rectal condition and its presence may be missed until the time of surgery. Radiological evaluation is essential for differential diagnosis of the clinically examined mass and the uncommon symptoms. As in our case, it was difficult to differentiate the actual origin of the giant mass with sonography Transvaginal clinical and sonographical examination was not possible. In transabdominal ultrasonography, the Y shape fundal indentation and depressed longitudinal groove extending from the uterus to the center of the inverted portion in every case is not recognized (4). MRI is the best radiological modality not only for the correct diagnosis, but also to delineate the lesion with neighbor structures (5). Computed tomography is not used as the first line examination in such pelvic masses, but it can be an option in conditions that MRI is not possible. Contrast enhanced examination is favored for delineation.

Standard pelvic MRI protocol with axial and sagittal T2-weighted scans revealed V-shaped uterine cavity and an inverted uterine fundus, and the uterine fundus was lined by a hyperintense endometrium surrounded by a hyperintense rim layering "bull's-eye" configuration with the adjacent mass. These images are the indicative signs for uterine inversion. As well as revealing the uterine inversion, magnetic resonance imaging can depict the stage of morphologic changes of the uterus, the ori- 
gin, extension, signal, and contrast enhancement properties of the mass, and the accompanying features of lesion invasion and pathological status of the lymph nodes.

A classification of genital inversion has been described and treatment of the inversion is suggested according to the stage and associated pathology. In stage 1, inversion of the uterus is intrauterine (incomplete) and the fundus remains within the cavity; stage 2 , complete inversion of the uterine fundus through the fibromuscular cervix; stage 3, total inversion; the fundus protrudes through the vulva; stage 4, the vagina is also involved within total inversion. While stage 1 , repositioning of the fundus could be tried for treatment, for stages 2-4, hysterectomy should be an option and should be discussed with the patient if childbearing is not an issue $(6,7)$. Eventually, radiologic evaluation is the key for stage definition and pre-operative decision-making. Radiologic algorithm should be followed from simple to detailed. Ultrasonography is the first-line evaluation method and MRI should be followed for insufficient and undetailed imaging as in our case. In the absence of a mass lesion, inversion is easily depicted in the transabdominal view. Transvaginal approach can be used for more complex cases in the early stages of the disease. Late stages of the disease and accompanying mass lesion to inversion often require MR imaging.

In conclusion, although being a rare entity, uterine inversion associated with giant pelvic mass can be easily recognized radiologically. If inversion occurs along with a giant mass, necrosis, infection, and severe anemia are evident and should be treated to avoid complications. The viability of the uterus is crucial for prognosis especially for younger patients. Therefore, the treatment should be arranged as soon as possible. This condition has to be clearly visualized for preoperative planning and also diagnostic suspicion of a malignant uterine tumor should be excluded. In our case, MRI was performed complementary to proper ultrasonographic evaluation. In this context, again, MRI is a valuable problem-solving tool for preoperative diagnosis of uterine inversion.

\section{Authors' Contributions}

Study concept: Veli Mihmanli and Fahrettin Kilic. Manuscript preparation: Fahrettin Kilic, Soner Pul, and Ahmet Kilickaya. Critical revision of the manuscript for important intellectual content: Veli Mihmanli, Fahrettin Kilic,and Aydin Kilinc.

\section{References}

1. Lupovitch A, England ER, Chen R. Non-puerperal uterine inversion in association with uterine sarcoma: case report in a 26-year-old and review of the literature. Gynecol Oncol. 2005;97(3):938-41.

2. Occhionero M, Restaino G, Ciuffreda M, Carbone A, Sallustio G, Ferrandina $G$. Uterine inversion in association with uterine sarcoma: a case report with MRI findings and review of the literature. Gynecol Obstet Invest. 2012;73(3):260-4.

3. Gomez-Lobo V, Burch W. Nonpuerperal uterine inversion associated with an immature teratoma of the uterus in an adolescent. Obstet Gynecol. 2008;112(3):708-9.

4. Buyukkurt S, Vardar MA, Zeren H, Ozgunen FT. Non-puerperal inversion of the uterus caused by leiomyosarcoma: a case report and clinical management. JObstet Gynaecol Res. 2007;33(3):402-6.

5. Moulding F, Hawnaur JM. MRI of non-puerperal uterine inversion due to endometrial carcinoma. Clin Radiol. 2004;59(6):534-7.

6. Sharma JB, Kumar S, Rahman SM, Roy KK, Malhotra N. Non-puerperal incomplete uterine inversion due to large sub-mucous fundal fibroid found at hysterectomy: a report of two cases. Arch Gynecol Obstet. 2009;279(4):565-7.

7. Auber M, Darwish B, Lefebure A, Ness J, Roman H. Management of nonpuerperal uterine inversion using a combined laparoscopic and vaginal approach. Am J Obstet Gynecol. 2011;204(6):e7-9. 